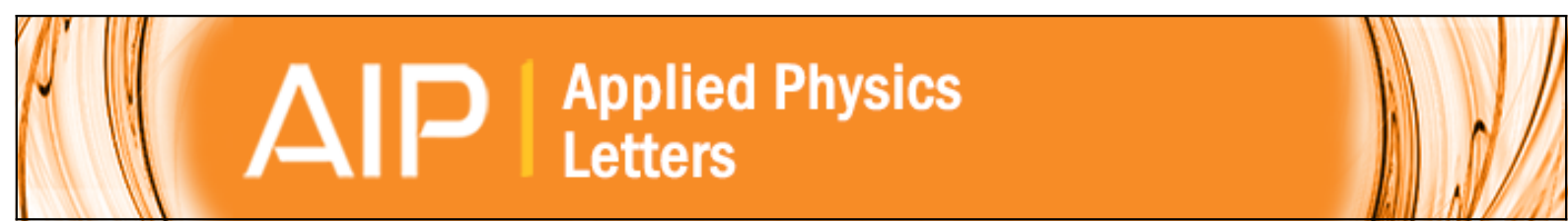

\title{
Near resonant and nonresonant third-order optical nonlinearities of colloidal InP/ZnS quantum dots
}

Y. Wang, X. Yang, T. C. He, Y. Gao, H. V. Demir, X. W. Sun, and H. D. Sun

Citation: Applied Physics Letters 102, 021917 (2013); doi: 10.1063/1.4776702

View online: http://dx.doi.org/10.1063/1.4776702

View Table of Contents: http://scitation.aip.org/content/aip/journal/apl/102/2?ver=pdfcov

Published by the AIP Publishing

\section{Articles you may be interested in}

Spectrally resolved size-dependent third-order nonlinear optical properties of colloidal CdSe quantum dots Appl. Phys. Lett. 100, 041102 (2012); 10.1063/1.3679381

Comparative photoluminescence study of close-packed and colloidal InP/ZnS quantum dots Appl. Phys. Lett. 96, 073102 (2010); 10.1063/1.3291049

Spectral and nonlinear optical characteristics of nanocomposites of $\mathrm{ZnO}-\mathrm{CdS}$

J. Appl. Phys. 103, 094914 (2008); 10.1063/1.2919109

Two-photon resonance assisted huge nonlinear refraction and absorption in $\mathrm{ZnO}$ thin films J. Appl. Phys. 97, 033526 (2005); 10.1063/1.1848192

Third-order optical nonlinearity in $\mathrm{ZnO}$ microcrystallite thin films Appl. Phys. Lett. 75, 3321 (1999); 10.1063/1.125338

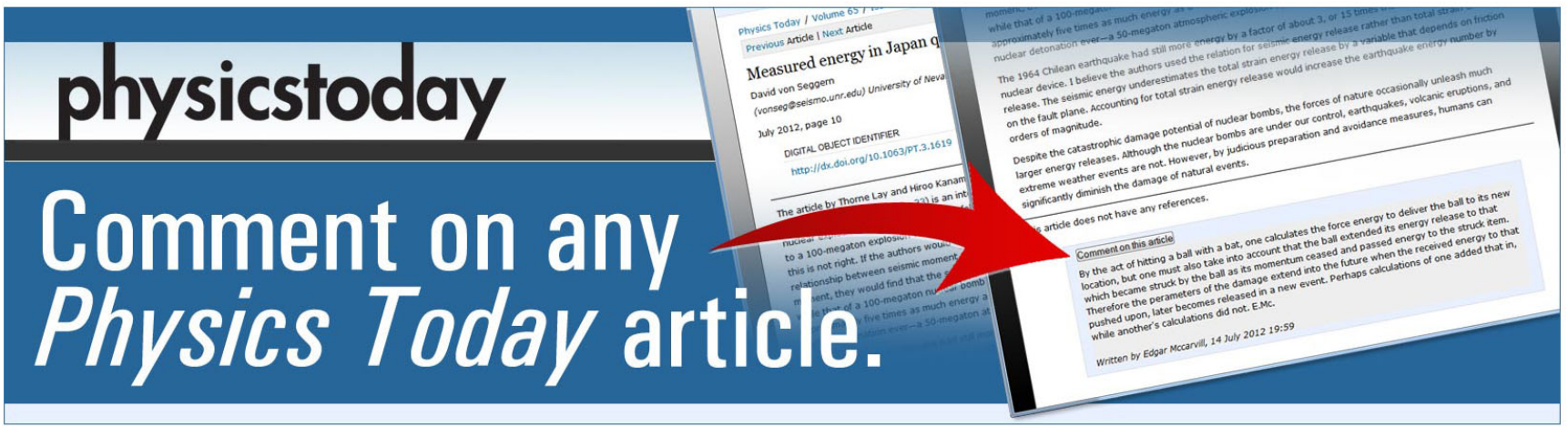




\title{
Near resonant and nonresonant third-order optical nonlinearities of colloidal InP/ZnS quantum dots
}

\author{
Y. Wang, ${ }^{1}$ X. Yang, ${ }^{2}$ T. C. He,${ }^{1}$ Y. Gao, ${ }^{1}$ H. V. Demir, ${ }^{1,2,3, a)}$ X. W. Sun,${ }^{2,4, a)}$ and H. D. Sun ${ }^{1,5, a)}$ \\ ${ }^{1}$ Division of Physics and Applied Physics, School of Physical and Mathematical Sciences, \\ Nanyang Technological University, Singapore 637371, Singapore \\ ${ }^{2}$ School of Electrical and Electronic Engineering, Nanyang Technological University, Nanyang Avenue, \\ Singapore 639798, Singapore \\ ${ }^{3}$ Department of Physics and Department of Electrical and Electronics Engineering, \\ UNAM-National Nanotechnology Research Center, Bilkent University, Ankara 06800, Turkey \\ ${ }^{4}$ South University of Science and Technology, Shenzhen 518055, China \\ ${ }^{5}$ Center for Disruptive Photonic Technologies (CDPT), Nanyang Technological University, Singapore
}

(Received 18 November 2012; accepted 2 January 2013; published online 18 January 2013)

\begin{abstract}
We have investigated the third-order optical nonlinearities of high-quality colloidal $\mathrm{InP} / \mathrm{ZnS}$ core-shell quantum dots (QDs) using Z-scan technique with femtosecond pulses. The two-photon absorption cross-sections as high as $6.2 \times 10^{3} \mathrm{GM}$ are observed at $800 \mathrm{~nm}$ (non-resonant regime) in $\mathrm{InP} / \mathrm{ZnS}$ QDs with diameter of $2.8 \mathrm{~nm}$, which is even larger than those of CdSe, CdS, and CdTe QDs at similar sizes. Furthermore, both of the $2.2 \mathrm{~nm}$ and $2.8 \mathrm{~nm}$-sized InP/ZnS QDs exhibit strong saturable absorption in near resonant regime, which is attributed to large exciton Bohr radius in this material. These results strongly suggest the promising potential of InP/ZnS QDs for widespread applications, especially in two-photon excited bio-imaging and saturable absorbing. (C) 2013 American Institute of Physics. [http://dx.doi.org/10.1063/1.4776702]
\end{abstract}

Semiconductor quantum dots (QDs) have attracted great interest for nonlinear photonic applications, including twophoton excited bio-imaging and saturable absorbing, thanks to their unique third-order nonlinear optical properties. ${ }^{1}$ Two-photon excited bio-imaging has several advantages compared to conventional one-photon excited bio-imaging, ${ }^{2}$ such as larger penetration depth into thick tissues, higher spatial resolution, and higher signal-to-noise ratio. As a result of their size-controlled absorption and emission spectra, large two-photon absorption (TPA) cross sections and high photostability, ${ }^{1}$ semiconductor QDs excel over the commonly used bio-imaging probes including organic dyes, ${ }^{3}$ fluorescent proteins, ${ }^{4}$ rare-earth doped nanoparticles, ${ }^{5}$ and semiconductor bulk crystal. ${ }^{6}$ Furthermore, semiconductor QD-based saturable absorbers exhibit much better performance than their quantum well and bulk counterparts owing to their large saturable absorption (SA) induced by quantum confinement effect. ${ }^{7} \mathrm{Up}$ till now, the third-order nonlinearities of II-VI QDs including CdSe, CdS, and CdTe QDs have been studied in detail. ${ }^{1,2,8}$ However, the inherent cytotoxicity of cadmium-based QDs casts doubt on their practical applications, especially in biological and biomedical areas. In contrast, indium phosphide (InP) QDs possess similar properties but without cytotoxicity due to the sturdiness of the covalent bond in III-V semiconductors instead of the ionic bond in the II-VI counterparts. ${ }^{9}$ A further advantage of InP QDs is the large exciton Bohr radius (15 nm) compared to that of CdS $(5.8 \mathrm{~nm})$, CdSe $(5.3 \mathrm{~nm})$, and CdTe $(7.3 \mathrm{~nm}),{ }^{10}$ which makes them promising for exhibiting strong SA in near resonant regime according to the theoretical analysis by Koch et al. ${ }^{11}$

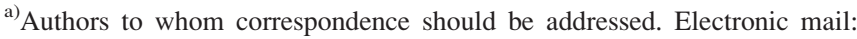
hvdemir@ntu.edu.sg,exwsun@ntu.edu.sg and hdsun@ntu.edu.sg.
}

Unfortunately, the much stronger coordinating strength of indium ligands than that of cadmium ligands makes the synthesis of high-quality colloidal InP QDs extremely difficult. ${ }^{12}$ Therefore, the previous reported InP QDs always possessed broad size distribution and low emission efficiency, and the optical studies of InP QDs were mostly limited within the linear part. ${ }^{9,13}$ Recently, by employing noncoordinating solvent and capping $\mathrm{InP}$ with $\mathrm{ZnS}$ shell of optimized thickness, high-quality InP/ZnS core-shell QDs were synthesized with a narrow size distribution and high emission quantum yield (QY) comparable to CdSe QDs. ${ }^{14}$ In this letter, we present our investigation of the optical nonlinearity on these QDs, and it is shown that the colloidal InP/ZnS QDs exhibit strong SA in near resonant regime and significant TPA in nonresonant regime indicating they are of great interest as nonlinear optical materials.

The detailed synthesis process of the high-quality colloidal InP/ZnS QDs can be found in Ref. 14. Figure 1 shows the one-photon excited photoluminescence (PL) and the UVvisible absorption spectra of two differently sized InP/ZnS QDs at room temperature. We denote them as QD606 and QD548, respectively, referring to their emission peak wavelengths. The diameters of QD548 and QD606 are estimated to be $2.2 \mathrm{~nm}$ and $2.8 \mathrm{~nm}$, respectively, based on their first exciton absorption peaks. ${ }^{15,16}$ The narrow emission linewidth and pronounced first exciton absorption peaks indicate that InP/ZnS QDs are uniform in size distribution. The inset in Figure 1 is the transmission electron microscopy (TEM) images of QD548 and QD606, which illustrate their good size uniformity. The QY was measured to be $58 \%$ and $39 \%$ for QD548 and QD606, respectively, relative to Rhodamine $6 \mathrm{G}$ in ethanol $(\mathrm{QY}=95 \%)$.

The single beam $\mathrm{Z}$-scan technique ${ }^{17}$ was utilized to measure the third-order nonlinearities of InP/ZnS QDs in 


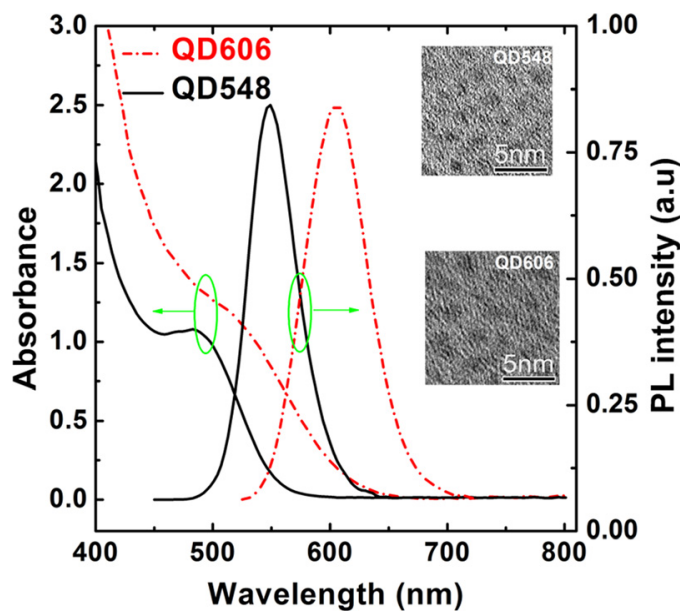

FIG. 1. UV-visible absorption spectra and normalized one-photon induced PL spectra of QD548 (2.2 nm) (black) and QD606 (2.8 nm) (red). The inset shows the corresponding TEM images of QD548 and QD606.

both near resonant and nonresonant regimes utilizing a femtosecond amplified-pulsed laser system with wavelength tunable. The laser pulses with pulse-width of $100 \mathrm{fs}$ and repetition rate of $1 \mathrm{KHz}$ were employed so that the thermal effects can be neglected. The validity of our Z-scan system was confirmed by performing calibration measurement with $\mathrm{CS}_{2}$ liquid, the nonlinear refractive index of $\mathrm{CS}_{2}$ was determined to be $3.5 \times 10^{-6} \mathrm{~cm}^{2} / \mathrm{GW}$, which was in accordance to the value measured by Ganeev et al. at similar conditions. ${ }^{28}$

The near resonant third-order nonlinearity of $\mathrm{InP} / \mathrm{ZnS}$ QDs was measured at $527 \mathrm{~nm}$ within the absorption band. Figure 2(a) shows the open aperture Z-scan curves of QD548 and QD606 at intensity of $5.5 \mathrm{GW} / \mathrm{cm}^{2}$. The peak-shape response indicates that $\mathrm{SA}$ or photoinduced transparency occurred in InP/ZnS QDs. Under the excitation of high power intensity, the available ground state carriers were depleted. On the other hand, the excited states became almost occupied, thus the Pauli exclusion principle came into play since QDs were similar to atoms or molecules. ${ }^{18}$ Therefore, the probability of optical transitions reduced significantly and SA occurred. The Z-scan theory ${ }^{17}$ was employed to fit the data to derive the nonlinear absorption coefficient $\alpha_{2}$. From the best-fit of the normalized transmittance, $\alpha_{2}$ is extracted to be $-0.59 \mathrm{~cm} / \mathrm{GW}$ (or $\operatorname{Im} \chi^{(3)}=-9.17 \times 10^{-13} \mathrm{esu}$ ) and $-1.93 \mathrm{~cm} / \mathrm{GW}$ (or Im $\chi^{(3)}=-28.01 \times 10^{-13} \mathrm{esu}$ ) for QD548 and QD606, respectively. Schrof et al. ${ }^{19}$ introduced $\left|\operatorname{Im} \chi^{(3)} / \alpha_{0}\right|$, where $\alpha_{0}$ is the linear absorption coefficient, as the figure of merit (FOM) to characterize saturable absorption property. We found that the FOM of $\left|\operatorname{Im} \chi^{(3)} / \alpha_{0}\right|$ for QD548 and QD606 are $8.0 \times 10^{-14}$ esu $\mathrm{cm}$ and $11.3 \times 10^{-14}$ esu $\mathrm{cm}$, respectively, both of which are larger than that of $15 \mathrm{~nm}$ gold nanoparticles $\left(3 \times 10^{-14} \mathrm{esu}\right.$ $\mathrm{cm}$ ) measured at the surface plasmon resonance (SPR). ${ }^{19}$

In order to derive the saturation intensity $I_{s}$ of $\mathrm{InP} / \mathrm{ZnS}$ QDs, the intensity-dependent Z-scan measurements were also performed, where the input intensity was tuned from $3.3 \mathrm{GW} / \mathrm{cm}^{2}$ to $37.5 \mathrm{GW} / \mathrm{cm}^{2}$. Figure 2(b) depicts the normalized transmittance change $\Delta T / T_{0}$, where $T_{0}$ is the linear transmittance at low intensity, as a function of input intensity $I_{0}$. This reveals that $\Delta T / T_{0}$ undergoes saturation at high input intensities. Similar phenomena have been observed in semiconducting single-walled carbon nanotubes (SWNTs) by
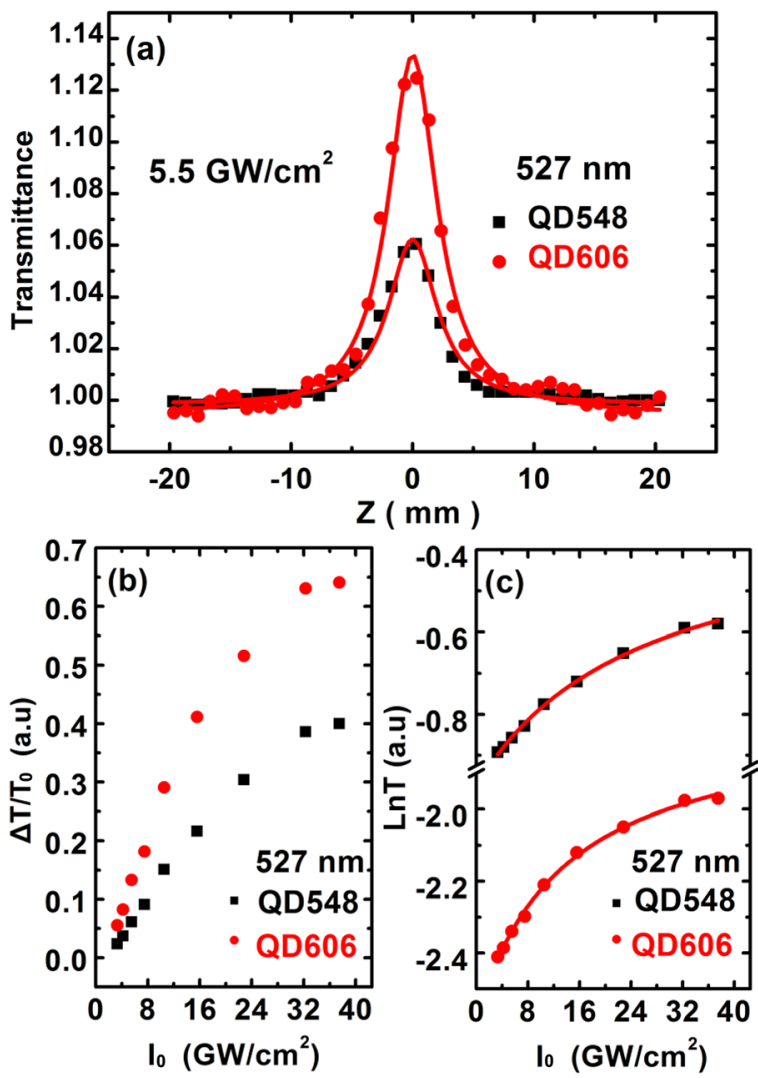

FIG. 2. (a) Open-aperture Z-scan curves of QD548 (ם) and QD606 (0) at the optical wavelength of $527 \mathrm{~nm}$ and the input intensity of $5.5 \mathrm{GW} / \mathrm{cm}^{2}$. The solid lines in (a) are the fits according to Z-scan theory. (b) Normalized transmittance change versus the input intensity tuning from 3.3 to $37.5 \mathrm{GW} /$ $\mathrm{cm}^{2}$ of QD548 (ם) and QD606 ( ). (c) A plot of natural logarithm of the transmittance at the focus plane in intensity-dependent Z-scan as a function of input intensity of QD548 (ם) and QD606 ( $)$.

Ostojic et al. ${ }^{20}$ In Figure 2(c), the natural logarithm of transmittance $T$ at the focus plane in the intensity-dependent $\mathrm{Z}$-scan measurement was plotted versus the input intensity $I_{0}$. From the best-fit of the data using formula $L n T=-\alpha_{0} L /$ $\left(1+I / I_{S}\right)$, where $\alpha_{0} /\left(1+I / I_{S}\right)$ is the intensity-dependent absorption coefficient ${ }^{21}$ and $L$ the optical path, we determined $I_{s}$ to be $19.8 \mathrm{GW} / \mathrm{cm}^{2}$ and $10.9 \mathrm{GW} / \mathrm{cm}^{2}$ for QD548 and QD606, respectively. These values were only slightly larger than that of SWNTs $\left(\sim 7 \mathrm{GW} / \mathrm{cm}^{2}\right),{ }^{20}$ which were already demonstrated to be good saturable absorbers. According to the theoretical analysis by Koch et al., ${ }^{11}$ the absorption change $\Delta \alpha$ or $\alpha_{2} I$, where $I$ is light intensity, of semiconductor QDs in near resonant regime is only proportional to the cube of the bulk exciton Bohr radius $\alpha_{e x}$ when the size of QDs and light intensity $I$ are fixed: $\Delta \alpha=24 \pi 10^{13}$ $\alpha_{e x}^{3} I /\left(c^{2} R^{3} \operatorname{Im} \tilde{\chi}_{3}\right)$, where $\mathrm{c}$ is the speed of light, $R$ is the radius of the QDs, and $\tilde{\chi}_{3}$ is the normalized third-order susceptibility which is independent of material parameters. Therefore, InP/ZnS QDs with large exciton Bohr radius $(15 \mathrm{~nm})$ make the best candidates for strong third-order nonlinearities as demonstrated in our experiments.

Thanks to their intrinsic cytotoxicity-free property, InP QDs are better bio-imaging probes than cadmium-based QDs. However, to date, the TPA cross-section of InP QDs has never been reported yet, which may be due to the low TPA and broad size distribution of InP QDs with poor quality. ${ }^{8}$ Here, the TPA cross-section and two-photon excited PL 
properties of $\mathrm{InP} / \mathrm{ZnS}$ QDs at $800 \mathrm{~nm}$, a widely used wavelength in two-photon excited bio-imaging, ${ }^{1}$ are reported. Figure 3(a) presents the two-photon excited PL spectra of QD548 and QD606 excited at $800 \mathrm{~nm}$, which are almost the same as the one-photon excited PL spectra indicating that the hot carriers under both one- and two-photon excitation relax to the same lowest exciton level, from which the radiative recombination occurs. ${ }^{22}$ The schematic diagram for the whole process of the one- and two-photon excited PL is illustrated in Figure 3(b). Similar phenomena were observed in CdSe (Ref. 22) and CdTe (Ref. 2) QDs. The corresponding twophoton excited PL images are shown as the inset in Figure 3(a). Figure 3(c) depicts the log-log plot of the PL intensities as a function of excitation intensity at $800 \mathrm{~nm}$, the nearly quadratic intensity dependence of the PL signals verifies the two-photon absorption induced PL process, rather than Auger-type upconversion PL. ${ }^{2}$ The open aperture Z-scan curves of QD548, QD606, and toluene at intensity of $45 \mathrm{GW} /$ $\mathrm{cm}^{2}$ are shown in Figure 4(d). The nearly flat response of toluene indicates that the nonlinearity of solvent was negligible and the nonlinear absorption response of QD548 and QD606
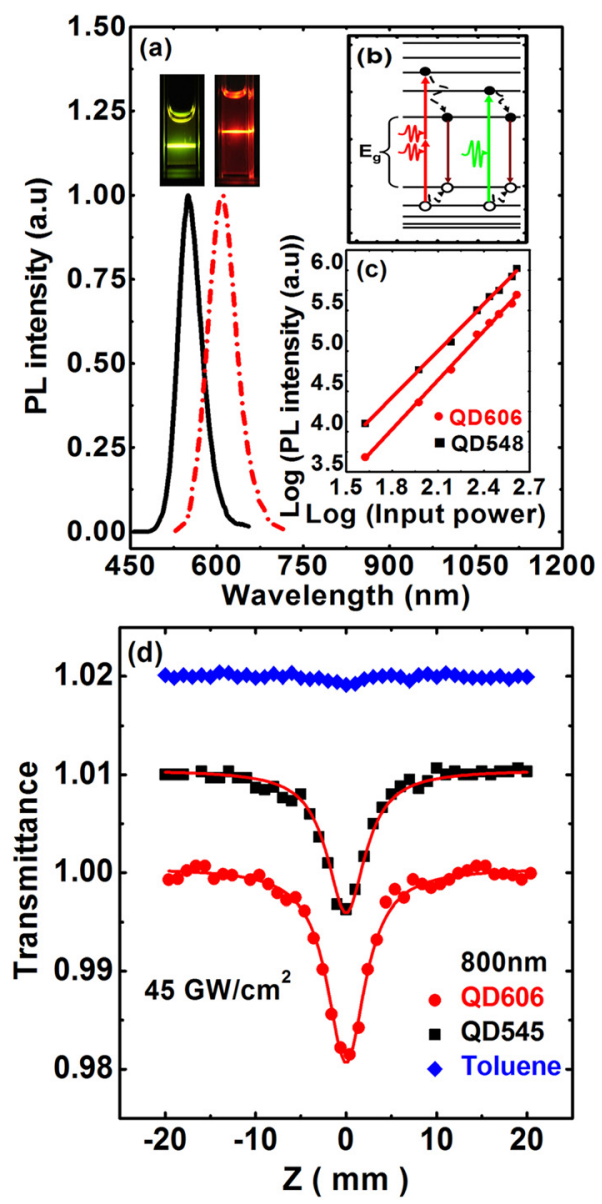

FIG. 3. (a) Normalized two-photon excited PL of QD548 (black) and QD606 (red) excited at $800 \mathrm{~nm}$. The corresponding two-photon excited PL images are shown in the inset. (b) Schematic diagram for the whole process of the one- and two-photon excited PL for QDs. (c) Log-log plots of the PL intensities as a function of excitation power at $800 \mathrm{~nm}$. The red lines are the linear fittings. The slopes for QD606 and QD548 are 2.03 and 1.93, respectively. (d) Open aperture Z-scan curves of QD548 ( $\mathbf{\square})$, QD606 (•), and toluene ( ) at the wavelength of $800 \mathrm{~nm}$ and the input intensity of $45 \mathrm{GW} / \mathrm{cm}^{2}$. The solid lines are the fitting curves using Z-scan theory. completely arose from the pure InP/ZnS QDs. The normalized transmittance of QD548 and QD606 was fitted well with the theoretical formula for TPA according to Z-scan theory. ${ }^{17}$ The TPA coefficients for QD548 and QD606 are extracted to be $0.009 \mathrm{~cm} / \mathrm{GW}$ and $0.012 \mathrm{~cm} / \mathrm{GW}$, respectively. The TPA cross-section $\sigma_{2}$ per $\operatorname{InP} / \mathrm{ZnS}$ QD can be deduced by: ${ }^{1}$ $\sigma_{2}=\beta h \gamma / N$, where $h \gamma$ is the photon energy and $N$ is the particle concentration in the solution. Here, $N$ is determined to be $6.6 \times 10^{19} / 1$ and $4.8 \times 10^{19} / 1$ for QD548 and QD606, respectively, employing the empirical relationship between the first exciton absorption peak, molar extinction coefficient, and size. ${ }^{16}$ The corresponding $\sigma_{2}$ are inferred to be 3.5 $\times 10^{-47} \mathrm{~cm}^{4}$ s photon ${ }^{-1}$ (or $3.5 \times 10^{3} \mathrm{GM}$ ) and $6.2 \times 10^{-47}$ $\mathrm{cm}^{4} \mathrm{~s}^{-4}$ photon $^{-1}$ (or $6.2 \times 10^{3} \mathrm{GM}$ ) for QD548 and QD606, respectively, which are comparable or even larger than that of CdSe $\left(5.1 \times 10^{3} \mathrm{GM}\right)$, CdTe $\left(2.1 \times 10^{2} \mathrm{GM}\right)$, and $\mathrm{CdS}$ $\left(4.4 \times 10^{3} \mathrm{GM}\right)$ QDs at similar sizes. ${ }^{1,8,23}$ Interestingly, the TPA cross-sections of CdS and CdSe QDs were found to increase with size, ${ }^{1,23}$ while those for $\mathrm{ZnS}$ and $\mathrm{ZnSe}$ QDs to decrease. ${ }^{24,25}$ The opposite trend of TPA cross-sections of QDs with size arises from the competition between decreased quantum confinement effect and increased density of state (DOS) when the sizes of QDs increase. ${ }^{1,25}$ The larger TPA cross-section of QD606 than that of QD548 indicates the increased DOS prevails over the decreased quantum confinement effect for InP/ZnS QDs. By taking into account the volume fraction and local field effect, the intrinsic TPA coefficient of $\mathrm{InP} / \mathrm{ZnS}$ QDs $\beta^{Q D}$ can be deduced by: ${ }^{23} \beta^{Q D}$ $=\beta /\left(f^{4} V_{f}\right)$, where $f=3 \varepsilon_{S} /\left(\varepsilon_{Q D}+2 \varepsilon_{s}\right)$ is the local-field factor, $\varepsilon_{s}$ is the dielectric constant of solvent, $\varepsilon_{Q D}$ is the dielectric constant of InP/ZnS QDs, and $V_{f}$ is the volume fraction of QDs relative to solvent. The dielectric constants $\varepsilon_{Q D}$ are inferred to be 9.72 and 9.92 for QD548 and QD606, respectively, using formula (15) in Ref. 10. The value of $\beta^{Q D}$ for QD548 and QD606 is calculated to be $516.9 \mathrm{~cm} / \mathrm{GW}$ and $487.3 \mathrm{~cm} / \mathrm{GW}$, respectively, which are greatly enhanced compared to that of bulk InP. ${ }^{26}$ The enhancement of TPA can be attributed to the enhanced oscillator strength induced by quantum confinement effect. ${ }^{11,18}$

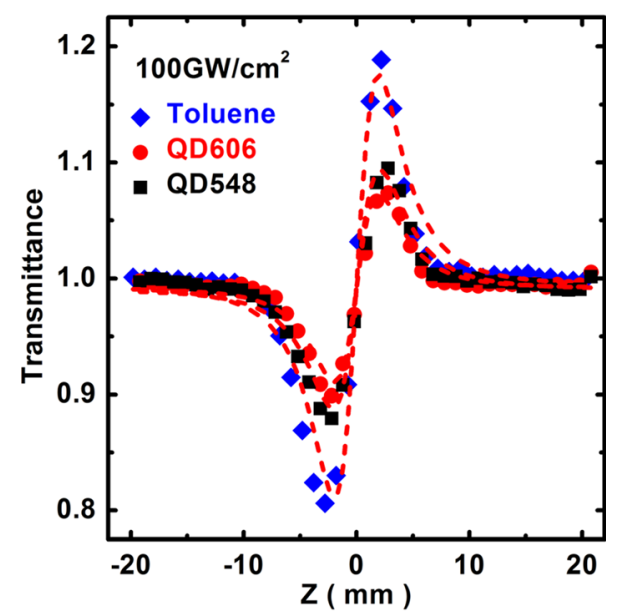

FIG. 4. CA/OA curves of Z-scan of QD548 (ם), QD606 (-), and toluene $(\diamond)$ at the wavelength of $800 \mathrm{~nm}$ and the input intensity of $100 \mathrm{GW} / \mathrm{cm}^{2}$. The dotted lines are the fitting curves using Z-scan theory. 
In addition, we found that the colloidal InP/ZnS QDs also exhibited high third-order nonlinear refraction. The third-order nonlinear refractive index of $\mathrm{InP} / \mathrm{ZnS}$ QDs at $800 \mathrm{~nm}$ was obtained by fitting the close aperture Z-scan curves divided by the corresponding open aperture Z-scan curves (CA/OA) so as to eliminate the influence of nonlinear absorption. Figure 4 shows the CA/OA curves of QD548, QD606, and pure toluene at intensity of $100 \mathrm{GW} / \mathrm{cm}^{2}$. The high intensity $\left(100 \mathrm{GW} / \mathrm{cm}^{2}\right)$ was chosen in order to magnify the difference between QD548 and QD606. The decreased magnitude of $\Delta T=T_{\text {Peak }}-T_{\text {Valley }}$ compared to pure toluene indicates the opposite signs of nonlinear refraction between InP/ZnS QDs and toluene. According to Z-scan theory, ${ }^{17}$ the third-order nonlinear refractive index $n_{2}$ of QD548, QD606, and toluene is determined to be $-0.57 \times 10^{-6} \mathrm{~cm}^{2} / \mathrm{GW}$, $-0.71 \times 10^{-6} \mathrm{~cm}^{2} / \mathrm{GW}$, and $1.3 \times 10^{-6} \mathrm{~cm}^{2} / \mathrm{GW}$, respectively. Similarly, when taking into account the volume fraction and local field effect, the intrinsic nonlinear refraction of $\mathrm{InP} / \mathrm{ZnS}$ QDs are given by: ${ }^{23} n_{2}^{Q D}=\left(n_{2}-\left(1-V_{f}\right)\right.$ $\left.n_{2}^{\text {sol }}\right) /\left(f^{4} V_{f}\right)$, where $n_{2}^{\text {sol }}$ is the nonlinear refractive index of toluene. $n_{2}^{Q D}$ are extracted to be $-3.1 \times 10^{-2} \mathrm{~cm}^{2} / \mathrm{GW}$ and $-2.9 \times 10^{-2} \mathrm{~cm}^{2} / \mathrm{GW}$ for QD548 and QD606, respectively, which are one order of magnitude larger than that of bulk InP. ${ }^{26}$ The large enhancement of third-order nonlinear refraction should result from the enhanced TPA because the dominant contribution to the third-order nonlinear refraction in nonresonant regime arose from the TPA term according to the analysis by Sheikbahae. ${ }^{27}$

In conclusion, the third-order nonlinearities of high quality colloidal InP/ZnS QDs were investigated. The intrinsic TPA is greatly enhanced compared to that of the bulk counterpart due to quantum confinement effect. Subsequently, the increased TPA gives rise to a significant enhancement of third-order nonlinear refraction. We also show that $\mathrm{InP} / \mathrm{ZnS}$ QDs with large exciton Bohr radius present strong SA within absorption band. Our results indicate that colloidal InP/ZnS QDs are extremely promising in a wide range of nonlinear optical applications.

This research was supported by the National Research Foundation of Singapore under its Competitive Research Programme (CRP Award No. NRF-CRP 6-2010-02), and the Singapore Ministry of Education through the Academic Research Fund (Tier 1) under the Project No. RG63/10. This work was also supported by Singapore Agency for Science,
Technology and Research (A*STAR) (Project Nos. 092101 0057 and 0921510088 ).

${ }^{1}$ X. Li, J. van Embden, J. W. M. Chon, and M. Gu, Appl. Phys. Lett. 94, 103117 (2009).

${ }^{2}$ A. Joly, W. Chen, D. McCready, J. Malm, and J. Bovin, Phys. Rev. B 71, 165304 (2005).

${ }^{3}$ T. C. He, P. C. Too, R. Chen, S. Chiba, and H. D. Sun, Chem. Asian J. 7, 2090 (2012).

${ }^{4}$ R. M. Dickson, A. B. Cubitt, R. Y. Tsien, and W. E. Moerner, Nature 388, 355 (1997).

${ }^{5}$ T. C. He, W. Wei, L. Ma, R. Chen, S. Wu, H. Zhang, Y. Yang, J. Ma, L. Huang, G. G. Gurzadyan, and H. D. Sun, Small 8, 2163 (2012).

${ }^{6}$ T. C. He, R. Chen, W. W. Lin, F. Huang, and H. D. Sun, Appl. Phys. Lett. 99, 081902 (2011).

${ }^{7}$ M. A. Cataluna, D. B. Malins, A. Gomez-Iglesias, W. Sibbett, A. Miller, and E. U. Rafailov, Appl. Phys. Lett. 97, 121110 (2010).

${ }^{8}$ B. H. Zhu, H. C. Zhang, J. Y. Zhang, Y. P. Cui, and Z. Q. Zhou, Appl. Phys. Lett. 99, 021908 (2011).

${ }^{9}$ D. J. Bharali, D. W. Lucey, H. Jayakumar, H. E. Pudavar, and P. N. Prasad, J. Am. Chem. Soc. 127, 11364 (2005).

${ }^{10}$ A. Franceschetti, H. Fu, L. W. Wang, and A. Zunger, Phys. Rev. B 60, 1819 (1999)

${ }^{11}$ L. Banyai, Y. Hu, M. Lindberg, and S. Koch, Phys. Rev. B 38, 8142 (1988).

${ }^{12}$ S. Xu, J. Ziegler, and T. Nann, J. Mater. Chem. 18, 2653 (2008).

${ }^{13}$ S. A. Gao, C. F. Zhang, Y. J. Liu, H. P. Su, L. Wei, T. Huang, N. Dellas, S. Z. Shang, S. E. Mohney, J. K. Wang, and J. A. Xu, Opt. Express 19, 5528 (2011).

${ }^{14}$ X. Y. Yang, D. W. Zhao, K. S. Leck, S. T. Tan, Y. X. Tang, J. L. Zhao, H. V. Demir, and X. W. Sun, Adv. Mater. 24, 4180 (2012).

${ }^{15}$ S. Adam, D. V. Talapin, H. Borchert, A. Lobo, C. McGinley, A. R. de Castro, M. Haase, H. Weller, and T. Moller, J. Chem. Phys. 123, 084706 (2005).

${ }^{16}$ P. Reiss, M. Protiere, and L. Li, Small 5, 154 (2009).

${ }^{17}$ M. Sheikbahae, A. A. Said, T. H. Wei, D. J. Hagan, and E. W. Vanstryland, IEEE J. Quantum Electron. 26, 760 (1990).

${ }^{18}$ A. P. Alivisatos, Science 271, 933 (1996).

${ }^{19}$ W. Schrof, S. Rozouvan, E. Van Keuren, D. Horn, J. Schmitt, and G. Decher, Adv. Mater. 10, 338 (1998).

${ }^{20}$ G. Ostojic, S. Zaric, J. Kono, M. Strano, V. Moore, R. Hauge, and R. Smalley, Phys. Rev. Lett. 92, 117402 (2004).

${ }^{21}$ S. H. Park, R. A. Morgan, Y. Z. Hu, M. Lindberg, S. W. Koch, and N. Peyghambarian, J. Opt. Soc. Am. B 7, 2097 (1990).

${ }^{22}$ G. S. He, K. T. Yong, Q. D. Zheng, Y. Sahoo, A. Baev, A. I. Ryasnyanskiy, and P. N. Prasad, Opt. Express 15, 12818 (2007).

${ }^{23}$ L. Y. Pan, N. Tamai, K. Kamada, and S. Deki, Appl. Phys. Lett. 91, 051902 (2007).

${ }^{24}$ V. V. Nikesh, A. Dharmadhikari, H. Ono, S. Nozaki, G. R. Kumar, and S. Mahamuni, Appl. Phys. Lett. 84, 4602 (2004).

${ }^{25}$ A. D. Lad, P. P. Kiran, D. More, G. R. Kumar, and S. Mahamuni, Appl. Phys. Lett. 92, 043126 (2008).

${ }^{26}$ M. D. Dvorak and B. L. Justus, Opt. Commun. 114, 147 (1995).

${ }^{27}$ M. Sheikbahae, D. C. Hutchings, D. J. Hagan, and E. W. Vanstryland, IEEE J. Quantum Electron. 27, 1296 (1991).

${ }^{28}$ R. A. Ganeev, A. I. Ryasnyansky, M. Baba, M. Suzuki, N. Ishizawa, M. Turu, S. Sakakibara, and H. Kuroda, Appl. Phys. B. 78, 433 (2004). 